Research Article

\title{
Fourier Rotman Lens Enabled Directional Modulation Transmitter
}

\author{
Yuan Ding, ${ }^{1}$ Yunhua Zhang, ${ }^{2}$ and Vincent Fusco ${ }^{1}$ \\ ${ }^{1}$ The Institute of Electronics, Communications and Information Technology (ECIT), Queen's University of Belfast, \\ Belfast BT3 9DT, UK \\ ${ }^{2}$ The School of Electronic Information, Wuhan University, Wuhan 430072, China
}

Correspondence should be addressed to Yuan Ding; yding03@qub.ac.uk

Received 26 November 2014; Accepted 1 March 2015

Academic Editor: Giampiero Lovat

Copyright (C) 2015 Yuan Ding et al. This is an open access article distributed under the Creative Commons Attribution License, which permits unrestricted use, distribution, and reproduction in any medium, provided the original work is properly cited.

\begin{abstract}
A $10 \mathrm{GHz}$ Fourier Rotman lens enabled dynamic directional modulation (DM) transmitter is experimentally evaluated. Bit error rate (BER) performance is obtained via real-time data transmission. It is shown that Fourier Rotman DM functionality enhances system security performance in terms of narrower decodable low BER region and higher BER values associated with BER sidelobes especially under high signal to noise ratio (SNR) scenarios. This enhancement is achieved by controlled corruption of constellation diagrams in IQ space by orthogonal injection of interference. Furthermore, the paper gives the first report of a functional dualbeam DM transmitter, which has the capability of simultaneously projecting two independent data streams into two different spatial directions while simultaneously scrambling the information signals along all other directions.
\end{abstract}

\section{Introduction}

Directional modulation (DM) technology, as a keyless physical layer security means, has developed in many aspects in recent years [1-25]. Generally speaking, DM enabled transmitter arrays have the capability of transmitting artificially distorted signals along all spatial directions other than an a priori specified communication direction in free space, in this way lowering the possibility of unwanted interception by eavesdroppers.

The characteristics of DM systems were mathematically described via a vector model presented in $[12,13]$. DM system performance assessment and cross system comparison metrics for DM system evaluation were established in [14], wherein DM systems were categorized as dynamic or static according to whether the array excitations for each unique transmitted symbol were updated at the transmitted data information rate or not. In $[4,5,14-19]$ several DM synthesis approaches, which placed emphasis on different DM system properties, for example, extra energy consumed [14], bit error rate (BER) spatial distributions $[4,5,15,16]$, far-field radiation patterns $[17,18]$, and interference spatial distributions [19], have been proposed. DM physical realization has been the subject of various studies. In [1-3] a DM architecture, which relies on the near-field coupling effects, was proposed. However, the deployment of a large number of parasitic array elements, as well as the unavailability of effective synthesis methods, may hinder its further development. Actively driven static DM arrays $[4-9,15]$ and antenna subset selection mechanisms [20-23] have been proposed. These DM architectures are synthesis-friendly, but all use reconfigurable devices in the radio frequency (RF) stage or at antenna level, for example, RF switches in [5, 20-23], and RF reconfigurable attenuators and phase shifters in $[4,6-8,15]$. These devices that have to operate at a speed much higher than the information rate require additional control and bias circuits and introduce loss. Critically no examples of physical dynamic DM transmitters have until this paper been reported.

There are two DM architectures that avoid the use of RF switchable devices and which are compatible, from the practical implementation perspective, with both static and dynamic DM systems. One is the digital DM architecture presented in [13]. The implementation of this digital structure requires careful system calibration, especially frequency and phase synchronization between each of the RF chains. Another promising DM architecture exploits the properties of Fourier 
transform beam-forming networks (FT-BFNs) or Fourier lens $[24,25]$. The beam orthogonality characteristic possessed by FT-BFNs [25] prevents the signals projected along the main information beam direction from being contaminated by interference injected at other beam ports of the Fourier lens. As was shown in [26], the additional phase adjustment could make classical Roman lens [27] exhibit Fourier transform function, enabling beam orthogonality in beam space. Furthermore, as pointed out in [24], when compared with the Butler matrix [28-30], the Fourier transform constrained Rotman lens [26] uses only two-dimensional (2D) printed circuit board (PCB) technology and is readily scalable by both frequency of operation and array port aperture size. Thus, we employ a Fourier Rotman lens to construct an experimentally evaluated dynamic DM transmitter.

Several system level experiments were conducted in an anechoic chamber. One is for general demonstration purpose, the video of which can be found in [31]. A real-time video stream with analogue amplitude modulation excited the 7 th beam port of a 13-by-13 Fourier Rotman lens, while the other ports were excited with $-25 \mathrm{~dB}$ sine-wave interference at $10 \mathrm{GHz}$, to yield a secure communication direction at boresight. In the video, it is observed that when compared with the conventional non-DM system, where the real-time video signal could be recovered in large spatial sectors, the Fourier Rotman DM system successfully confined the decodable region into a small area around boresight. The second experiment employed the Wireless Open-Access Research Platform (WARP) [32], which allowed digital modulation characteristics to be evaluated. This experiment forms the basis for this paper and the experimental setup used is described in Section 2 , leaving experiment results and discussion presented in Section 3. Conclusions are drawn in Section 4.

\section{Experimental Setup}

A 13-by-13 Fourier Rotman lens DM transmitter for $10 \mathrm{GHz}$ operation was built according to the design rules in [24] and experiments were conducted for real-time data transmission in a $10 \times 6 \times 6 \mathrm{~m}^{3}$ anechoic chamber.

The block diagram of the Fourier Rotman lens DM experimental setup is illustrated in Figure 1, and photographs are shown in Figure 2. Here WARP boards are used for modulation, demodulation, and BER measurement.

The workflow for experimental characterization of the Fourier Rotman lens DM system is now presented.

(a) Connect some or all of the data carrying ports RF1, RF2, and RF3; see Figures 1 and 2, to the input ports (beam ports) of the Fourier Rotman lens, according to the different system configurations that will be discussed in Section 3.

(b) Rotate the DM transmit antenna array, in azimuth for a one-dimensional (1D) array, in order to align the receive horn antenna along a spatial direction $\theta$ $\left(\theta \in\left[0^{\circ}, 180^{\circ}\right]\right)$ of interest.

(c) Initialize the WARP nodes a and $\mathbf{b}$, Figures 1 and 2. This includes the following. (i) Allocate RF ports numbers. To facilitate discussion, they are labeled as RFa to RFd ( $R F a, R F b$, and $\mathrm{RFC}$ on transmit node $\mathrm{a}, \mathrm{RFd}$ on receive node $\mathbf{b})$.

(ii) Set operation frequency. $2.437 \mathrm{GHz}$ (channel 6 in the $2.4 \mathrm{GHz}$ ISM band) was used in the experiment.

(iii) Set transmitter and receiver gains.

(d) Download data samples that are generated in MAT$\mathrm{LAB}[33]$ on a PC into transmit buffers in the FPGA on the WARP board a. Data applied to ports RFa and $\mathrm{RFb}$ are modulated for DQPSK with $2.5 \mathrm{Msps}$ symbol rate and for DBPSK with $5 \mathrm{Msps}$ symbol rate, respectively, unless otherwise specified. Differential modulation schemes are chosen because this simplifies demodulation at the receive side. The same Barker sequence preambles modulated with BPSK are appended ahead of the data in order to identify where the data begins in the receive buffer after signals are captured at the receive side. Orthogonal interference signals are applied via RFc.

(e) Send triggers to the WARP nodes $\mathbf{a}$ and $\mathbf{b}$. When the triggers are received, the data samples in FPGA transmit buffers on WARP board a are passed into digital to analogue converters (DACs) before being upconverted to $2.437 \mathrm{GHz}$ by the transceiver MAX2829 [34] and further upconverted to $10 \mathrm{GHz}$ by the mixer HMC220AMS8E [35] and finally distributed to the patch antenna array through the 13by-13 Fourier Rotman lens. The transmitted signals along $\theta$, captured by the receive horn antenna, are frequency downconverted by mixer HMC220AMS8E and then MAX2829 and digitized through analogue to digital converters (ADCs) and finally stored in the receive buffer on the WARP board $\mathbf{b}$. This raw data is read into MATLAB for analysis. In order to achieve the frequency synchronization between the transmitter and the receiver, a common $80 \mathrm{MHz}$ sampling reference clock and RF reference clock for both WARP boards a and $\mathbf{b}$ are provided externally by the AWG5012. However, the signal generators Agilent 83650B and Rohde\&Schwarz SMR20 used to generate RF continuous waves LO1, LO2, and LO3, all at $7.563 \mathrm{GHz}$, for upconversion and downconversion, are frequency synchronized by sharing a common $10 \mathrm{MHz}$ reference clock; see Figure 1.

(f) Artificial AWGN is added onto the received raw data in MATLAB to facilitate various SNR scenarios. The system intrinsic AWGN is sufficiently low to be ignored. This was validated through an experiment where zero BERs after $10^{+6}$ data bits transmissions were detected along any spatial direction in a conventional non-DM system positioned in the anechoic chamber.

(g) Iterate steps (d) to (f) until the required number of data bits is transmitted. $10^{+6}$ was used in the experiment. The BER along $\theta$ can then be computed. 


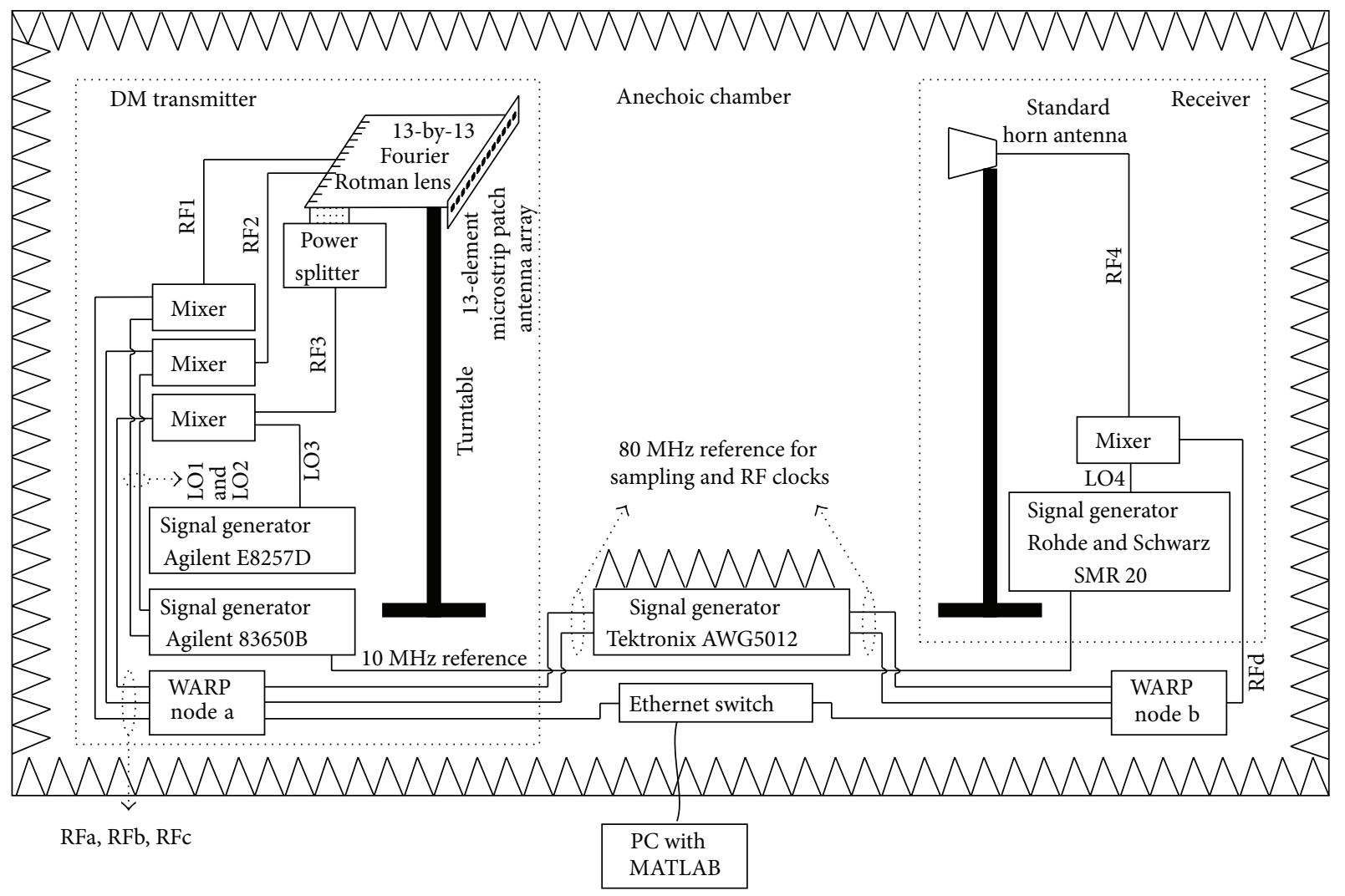

FIGURE 1: Block diagram of the 13-by-13 Fourier Rotman lens DM experiment setup.
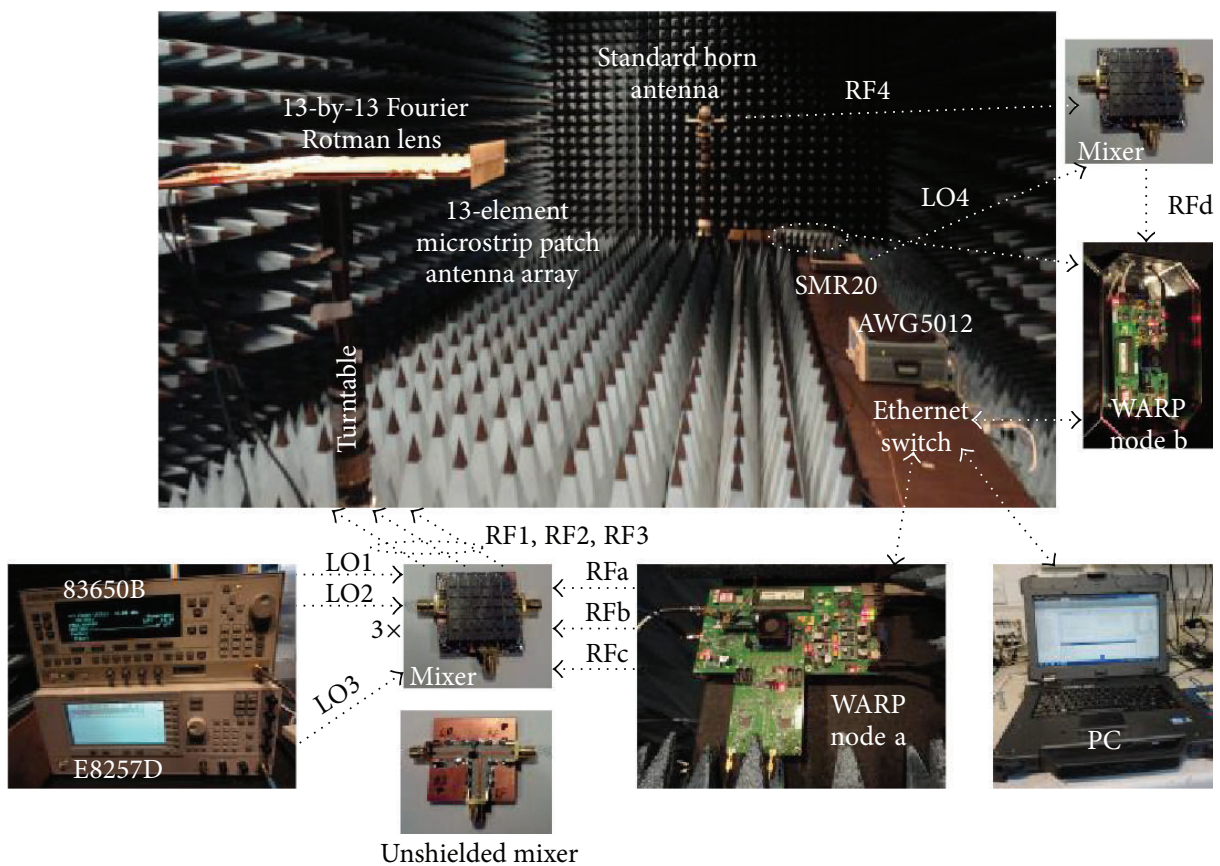

Figure 2: Photographs of the 13-by-13 Fourier Rotman lens DM experiment. 


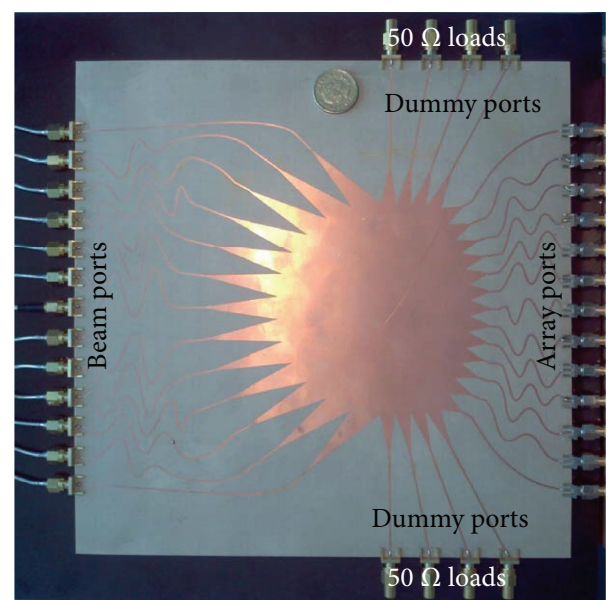

FIGURE 3: Fabricated 13-by-13 Fourier transform constrained Rotman lens for $10 \mathrm{GHz}$ operation.

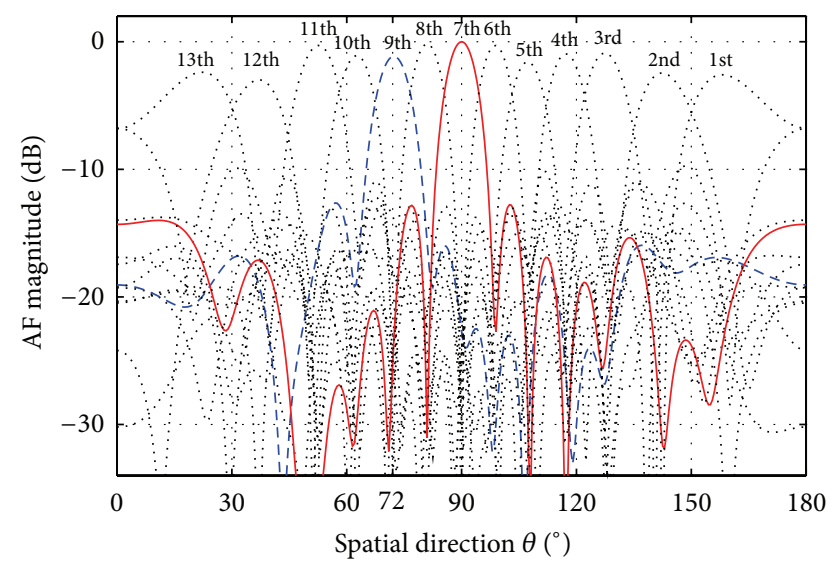

Figure 4: Array factors (AFs) for each beam port excited separately based on measured Fourier Rotman lens $S$-parameters. The AFs for the 7 th and 9th beam port excitations are highlighted.

(h) $\operatorname{Scan} \theta$ within $0^{\circ}$ to $180^{\circ}$ and repeat steps (c) to (g). BER spatial distribution is obtained under prespecified system configurations and SNR conditions.

Before presenting sample experimental results in Section 3 , the characteristics of the 13-by-13 Fourier Rotman lens and the 1D 13-element microstrip patch antenna array for $10 \mathrm{GHz}$ operation, used in the experiment, are now presented.

2.1. 13-by-13 Fourier Rotman Lens at $10 \mathrm{GHz}$. A 13-by-13 Fourier transform constrained Rotman lens [26] for $10 \mathrm{GHz}$ operation was designed and fabricated on a substrate of Taconic RF-60A [36], thickness $0.64 \mathrm{~mm}$, and relative dielectric constant 6.15 , Figure 3 . The overall board dimension, including the extended $50 \Omega$ microstrip lines, is $250 \mathrm{~mm} \times$ $250 \mathrm{~mm}$. Different to the Fourier Rotman lens used in [24], the array ports are aligned with half wavelength spacing, which enables direct connection between the lens and the microstrip patch antenna array, eliminating cable induced magnitude and phase errors. Using measured $S$-parameters

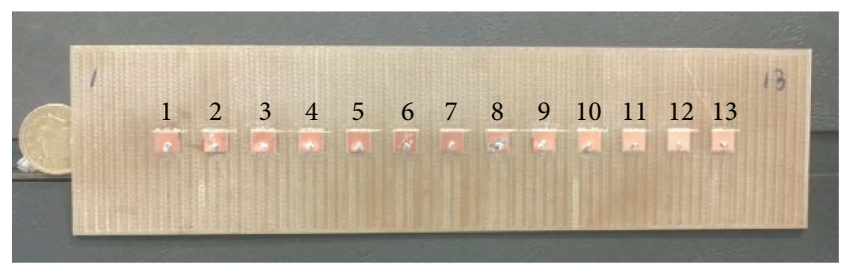

FIGURE 5: Front view of the fabricated 1D 13-element microstrip patch antenna array for $10 \mathrm{GHz}$ operation. A ground plane covers the whole of the back of the board.

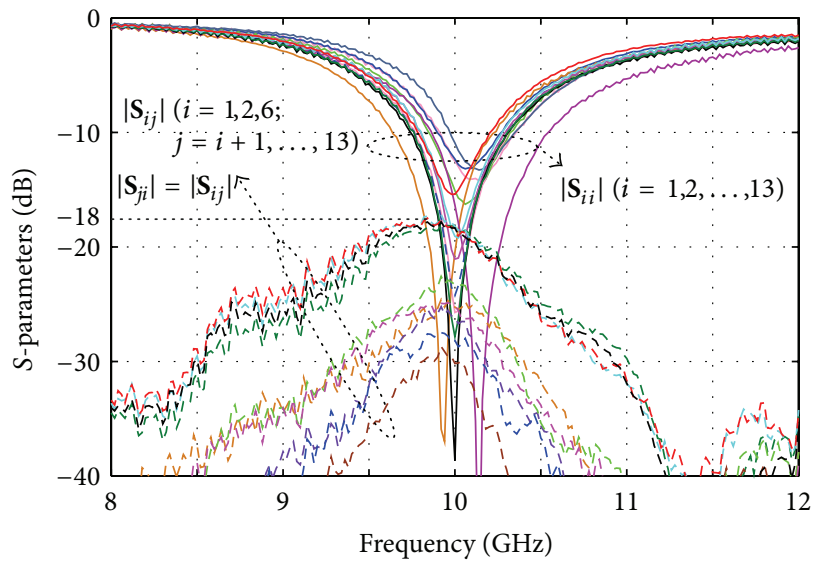

FIGURE 6: Measured S-parameters of the fabricated 1D 13-element microstrip patch antenna array.

at $10 \mathrm{GHz}$, the normalized array factors (AFs) for each beam port excited separately were computed and are presented in Figure 4. The beam orthogonality properties of the lens can be observed; for example, along the directions of the 7 th and the 9 th beams, $90^{\circ}$ and $72^{\circ}$, the interference beam leakage from other beam ports is below $-20 \mathrm{~dB}$ and $-14 \mathrm{~dB}$, respectively. The fabrication errors and nonuniform insertion loss between each beam port and array port pair contribute to the nonorthogonality, particularly for beams away from boresight.

2.2. 1D 13-Element Microstrip Patch Antenna Array. A 1D 13element microstrip patch antenna array with half wavelength spacing for $10 \mathrm{GHz}$ operation was designed and fabricated on the FR4 substrate [37], thickness $1.57 \mathrm{~mm}$, and relative dielectric constant 4.7 , Figure 5 . The fabricated antenna array is $240 \mathrm{~mm}$ in length and $60 \mathrm{~mm}$ in width.

The $S$-parameters of the patch antenna array were measured and are depicted for the frequency range from $8 \mathrm{GHz}$ to $12 \mathrm{GHz}$ in Figure 6. It can be observed that at $10 \mathrm{GHz}$ array elements are well matched $\left(\left|\mathbf{S}_{i i}\right|<-11 \mathrm{~dB}, i=1,2, \ldots, 13\right)$, and cross coupling is less than $-18 \mathrm{~dB}$.

The far-field active element pattern (AEP) [38], both magnitude and phase in the azimuth plane of each antenna element, was measured in an anechoic chamber and is presented in Figure 7. Here we normalize the measured power patterns with respect to the magnitude value of the center element, that is, antenna 7 , along boresight $\left(90^{\circ}\right)$ to 


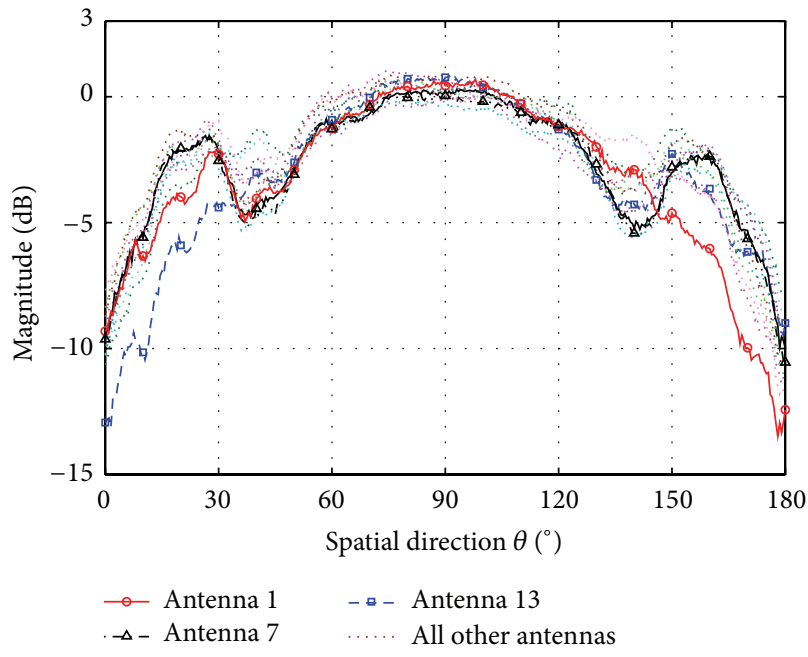

(a)

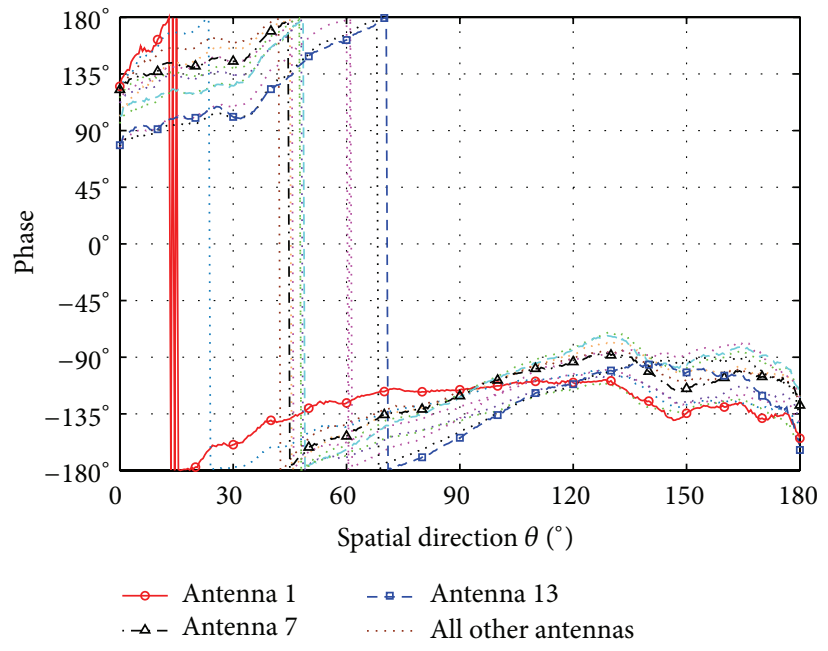

(b)

FIGURE 7: Measured AEP, (a) normalized magnitude and (b) phase, of each antenna element in the array. Curves for the antennas 1, 7, and 13 are highlighted.

$0 \mathrm{~dB}$. During phase pattern measurement, the antenna test rotator axis and the geometric center of each corresponding patch antenna element were aligned.

The far-field radiation pattern for each Fourier Rotman lens beam port excitation when the array ports are connected to the antenna array is shown in Figure 8. Compared with the AFs in Figure 4, along the direction of the main beam of each pattern in Figure 8, there is additional leaked interference energy caused by the remaining patterns. This is due to the nonidentical AEP of each antenna element; see Figure 7, particularly along the spatial angles further away from boresight. However, it can be seen that more than $10 \mathrm{~dB}$ difference between the $i$ th $(i=3,4,6,7, \ldots, 11)$ main beam and leaked energy from other patterns can still be obtained.

\section{Experimental Results and Discussion}

In this section, the Fourier Rotman lens DM experimental results are provided. Firstly, the experimental setup is validated by comparing measured BER results with simulated BER results that are calculated based on the measured farfield radiation patterns in Figure 8 for a conventional beamsteering array, that is, one where no interference is injected into the system. Secondly, the orthogonality between the information and the interference in the beam space is checked by injecting interference under relatively low SNR scenarios. Next, results that show DM spatial BER advantages over the conventional array are presented under high SNR conditions. Finally, some experimental results on the properties of a dualbeam DM system where two separate information beams are projected simultaneously into two different directions are presented.

3.1. Validation of Experimental Setup and BER Measurement. In Figure 9(a), the measured BER spatial distribution and the calculated one that is based on the measured patterns

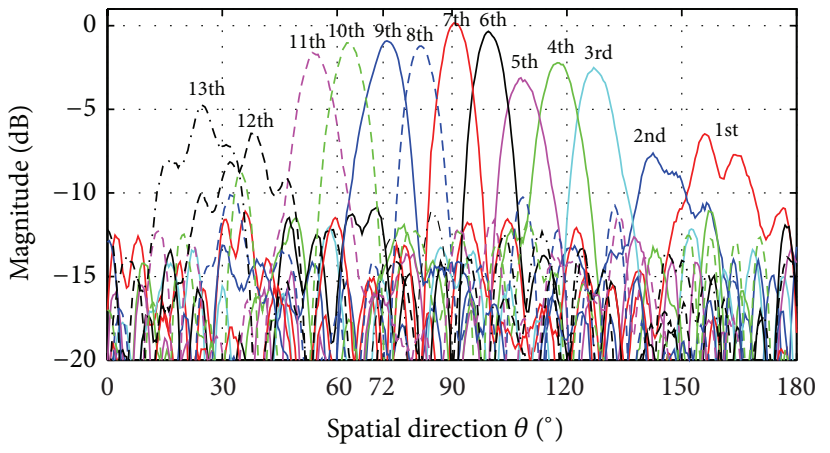

FIGURE 8: Normalized far-field radiation pattern for each Fourier Rotman lens beam port excitation when the array ports are connected to the microstrip patch antenna array. The far-field patterns for the 7 th and 9 th beam port excitations are highlighted.

in Figure 8 are depicted. At the transmit side, only RF1 was used for data transmission, while RF2 and RF3 were disabled. This makes the transmitter a conventional beam-steering array. In the experiment $10^{+6}$ random DQPSK symbols with $2.5 \mathrm{Msps}$ symbol rate were injected into the 7 th beam port of the Fourier Rotman lens; that is, the main BER beam was projected along boresight direction. The power of the artificial AWGN added onto the received raw data in MATLAB was adjusted to make the BER along $90^{\circ} 10^{-4}$, which corresponds to a SNR of approximate $13.8 \mathrm{~dB}$. When the SNR was increased to $25.8 \mathrm{~dB}$, which was achieved by decreasing the applied AWGN, the BER results obtained are shown in Figure 9(b).

For further validation similar BER measurement and simulation were conducted with the 9th beam port excited with $10^{+6}$ random DBPSK data signals; see Figure 10. Here at the transmit side only RF2 was used. In order to normalize the BER value along $72^{\circ}$ to $10^{-4}$, the SNR was adjusted to $9.2 \mathrm{~dB}$. 


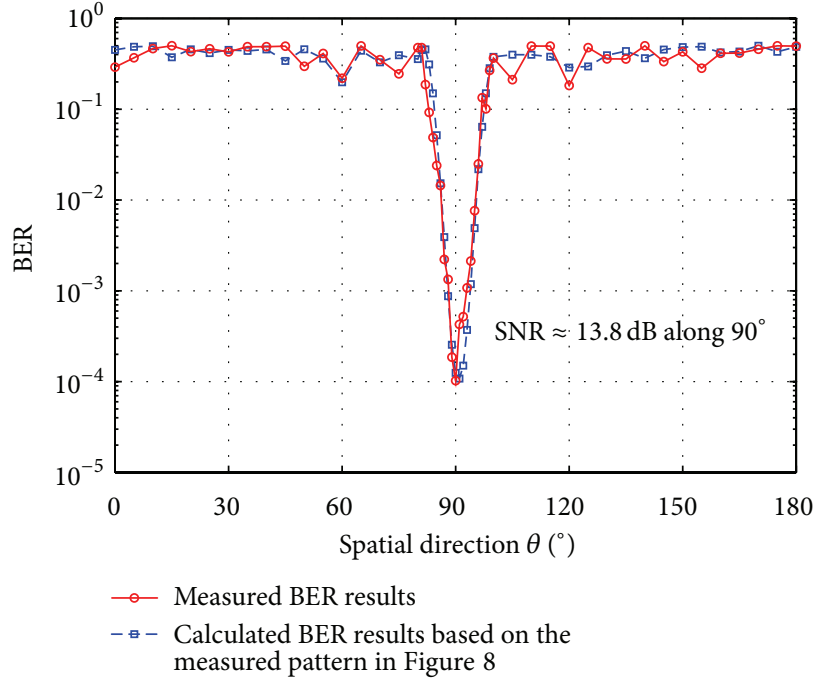

(a)

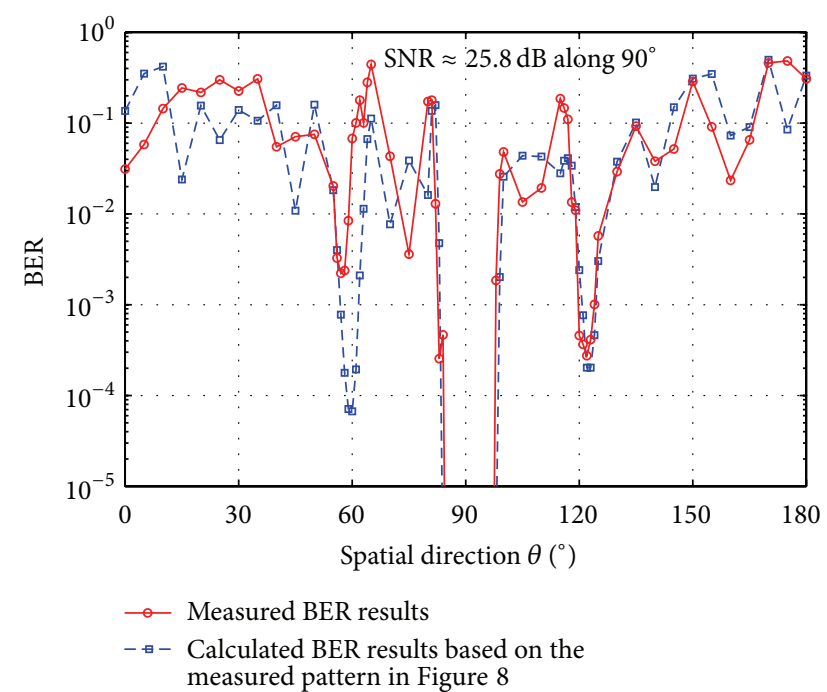

(b)

FIGURE 9: Measured and calculated BER spatial distributions based on the measured pattern in Figure 8 under $13.8 \mathrm{~dB}$ and $25.8 \mathrm{~dB}$ SNRs. Only the 7th beam port of the Fourier Rotman lens was excited with DQPSK data streams.

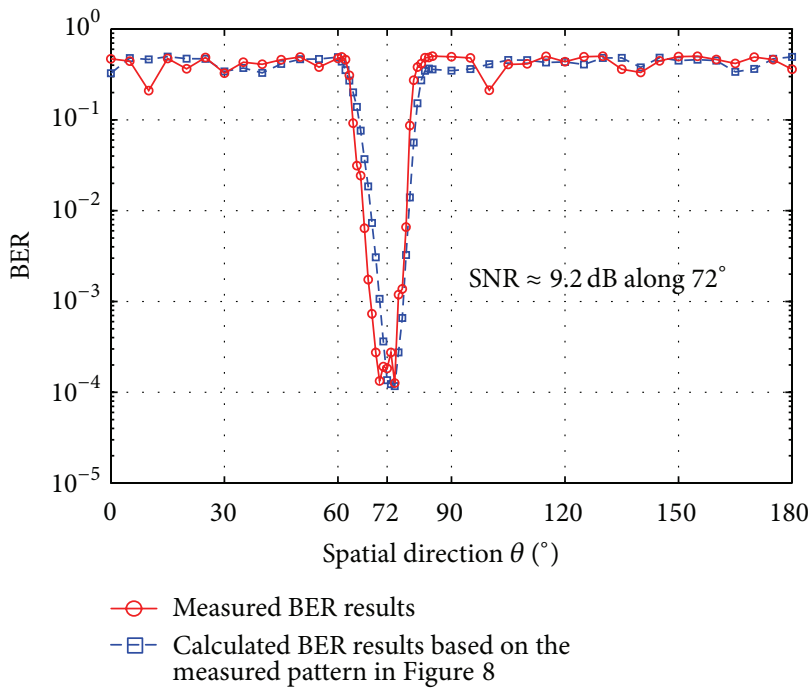

(a)

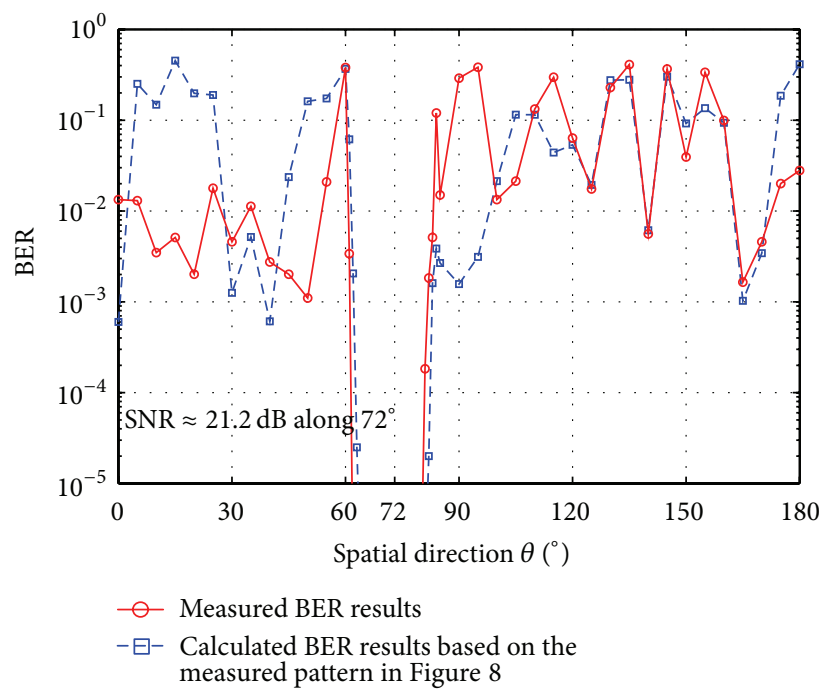

(b)

FIGURE 10: Measured and calculated BER spatial distributions based on the measured pattern in Figure 8 under $9.2 \mathrm{~dB}$ and $21.2 \mathrm{~dB}$ SNRs. Only the 9th beam port of the Fourier Rotman lens was excited with DBPSK data streams.

From Figures 9 and 10, it can be seen that the measured BER results well resemble their simulated counterparts, validating the experimental setup and BER measurement. In order to facilitate the discussion about the effects of the injected artificial noise on the information signal in the remaining section, the power normalized detected constellation diagrams in IQ space along $90^{\circ}$ and $72^{\circ}$, respectively, for DQPSK and DBPSK cases are shown in Figure 11. The SNRs are $13.8 \mathrm{~dB}$ and $9.2 \mathrm{~dB}$, respectively. We observe that the constellation diagrams along other directions are similar, but each symbol cluster is more spread due to lower signal power projected.
3.2. Validation of Orthogonality between Information and Interference in Beam Space. The orthogonality between information and interference in beam space was checked by measuring BERs with and without interference injected. In order to route artificial interference to the remaining 12 beam ports of the Fourier Rotman lens, a 1-to-16 Wilkinson power splitter for $10 \mathrm{GHz}$ operation was designed and built on FR4 substrate [37]; see Figure 12. Only 12 output ports were used in the experiment.

The interference routed to the remaining 12 beam ports of the Fourier Rotman lens was generated by the RF3 on the WARP node a. This was kept at a constant magnitude, 


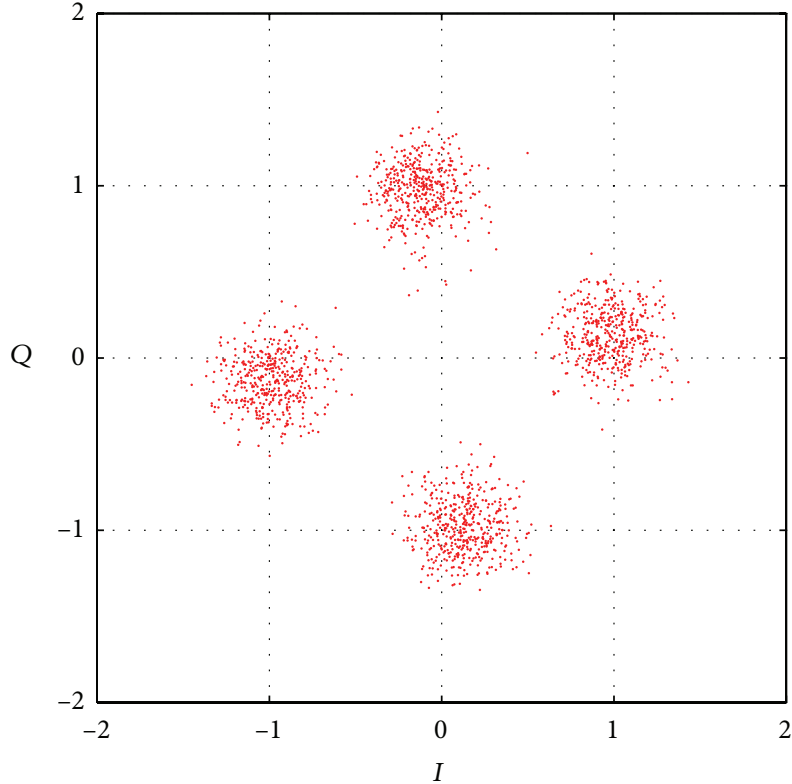

(a)

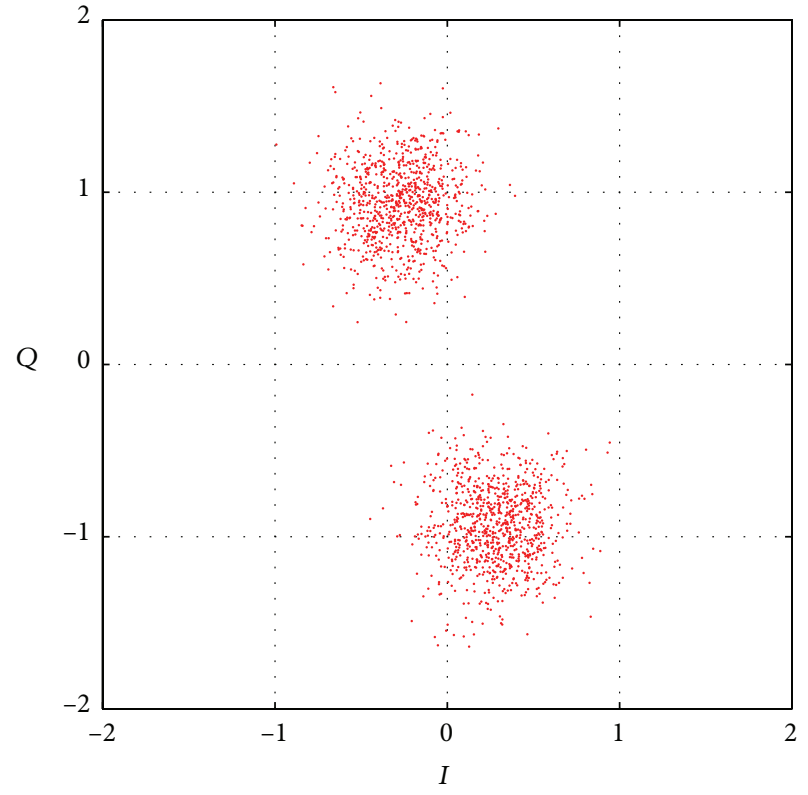

(b)

FIGURE 11: Power normalized detected (a) DQPSK and (b) DBPSK constellation diagrams in IQ space along $90^{\circ}$ and $72^{\circ}$, when the 7 th and the 9th beam ports of the Fourier Rotman lens were excited with DQPSK and DBPSK signals, respectively. The SNR for each case was set to $13.8 \mathrm{~dB}$ and $9.2 \mathrm{~dB}$.

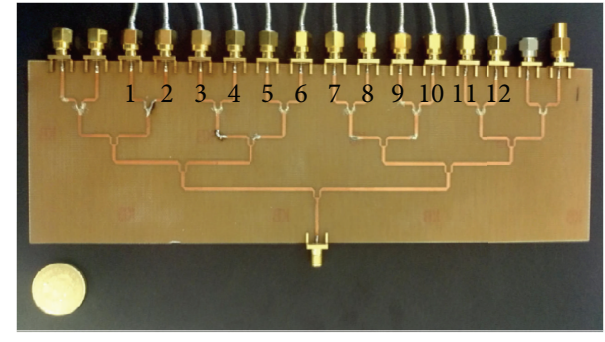

Figure 12: Fabricated 1-to-16 Wilkinson power splitter for $10 \mathrm{GHz}$ operation. Only output ports marked 1 to 12 were used in the experiment.

either -5 or $0 \mathrm{~dB}$ at each interference port relative to the information signal power, but was updated with random phases selected within the range $0^{\circ}$ to $360^{\circ}$ at the symbol rate. The magnitude of interference was chosen to be constant in order to minimize the nonlinearity that active devices may introduce. In Figure 13 we present the measured BER results for the cases of DQPSK and DBPSK information signals, which were applied at the 7th and the 9th beam ports, respectively and alternately. It can be observed that the interference injected into the system has the capability of narrowing the decodable spatial region. Also it has little effect on the detected BERs along the selected communication directions, $90^{\circ}$ and $72^{\circ}$ for each case, which indicates that the orthogonality between information and interference in beam space is being achieved by the Fourier Rotman lens.

3.3. DM System Performance under High SNR Scenarios. In order to better visualize the secrecy performance advantages of DM systems over conventional non-DM systems, the BER spatial distributions were obtained under high SNR scenarios, $25.8 \mathrm{~dB}$ for the DQPSK transmitters with main beam towards boresight and $21.2 \mathrm{~dB}$ for the DBPSK transmitters with main beam projected along $72^{\circ}$. Instead of applying interference at each of the remaining 12 beam ports, only two ports next to the information ports were excited with interference, that is, the 6th and the 8th ports for the DQPSK case and the 8th and the 10th ports for the DBPSK case. These ports were selected because they project main interference beams closely around the main information beam, which narrows the decodable spatial region in an energy efficient manner.

Figure 14 illustrates the measured BER spatial distributions for the Fourier Rotman lens DQPSK and DBPSK DM systems for various interference configurations. It can be concluded that, compared with the conventional Fourier Rotman lens beam-steering systems where no interference is injected and whose BER responses are also provided in Figure 14, the DM system here exhibits narrower main BER beamwidth and higher BER values associated with BER sidelobes. Consequently the more the interference power, the more enhanced the secrecy performance that a DM system can achieve.

In order to further investigate how injected orthogonal interference affects signal formats along unselected spatial directions, the detected constellation diagrams for the DQPSK systems along $122^{\circ}$, where a noticeable BER sidelobe exists in the conventional system (see Figure 14(a)) under SNR of $25.8 \mathrm{~dB}$ are depicted in Figure 15. It can be observed that a typical DQPSK constellation pattern was detected when no interference was injected, Figure 15(a). With the interference of constant magnitude and random phase, each constellation symbol cluster spreads into a circle, 


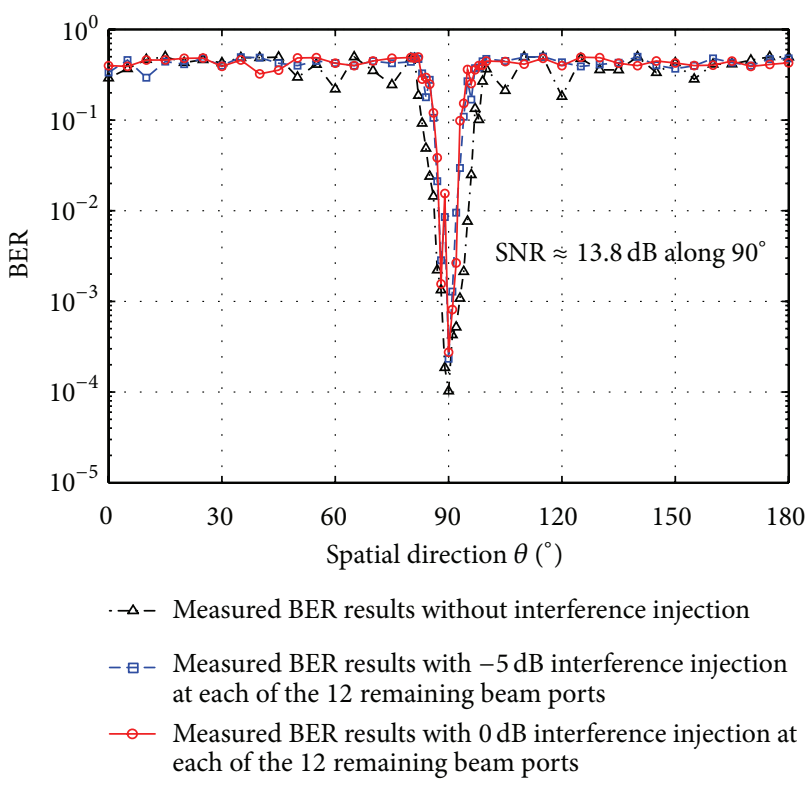

(a)

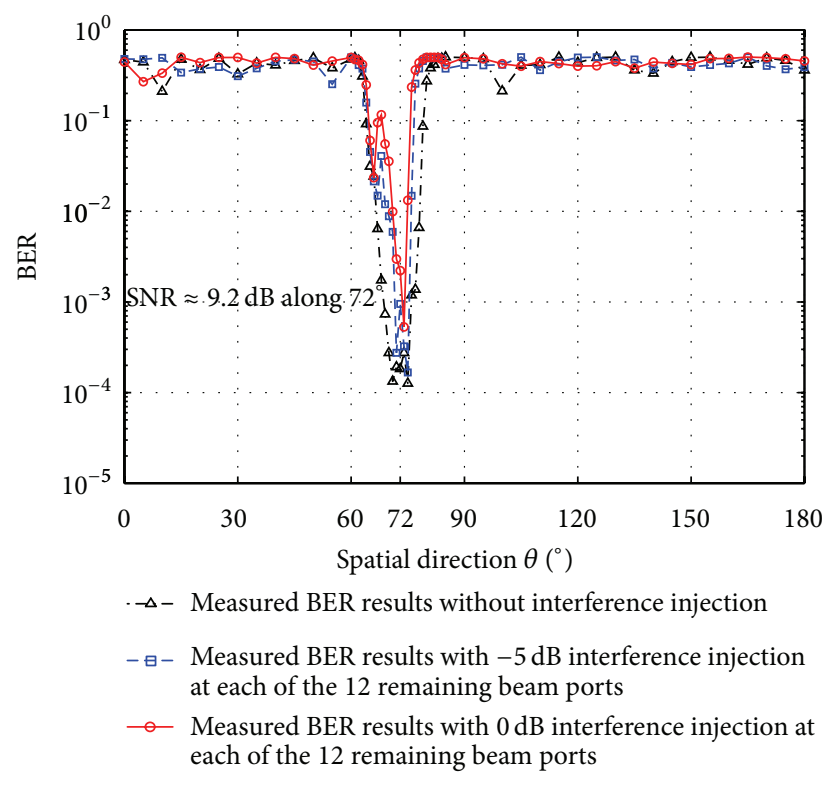

(b)

FIGURE 13: Measured BER results for the cases of (a) DQPSK and (b) DBPSK information signals, which were applied at the 7th and the 9th beam ports of the Fourier Rotman lens, respectively and alternately. Interference with $-\infty,-5$, or $0 \mathrm{~dB}$ magnitude and symbol rate updated random phases was injected into each of the remaining 12 beam ports. The SNR for each case was set to $13.8 \mathrm{~dB}$ and $9.2 \mathrm{~dB}$.

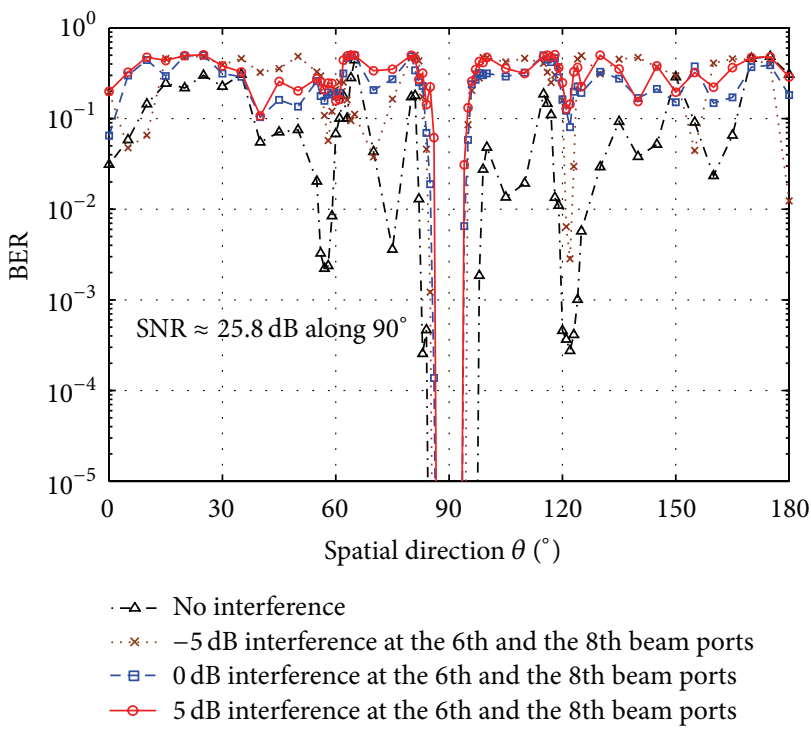

(a)

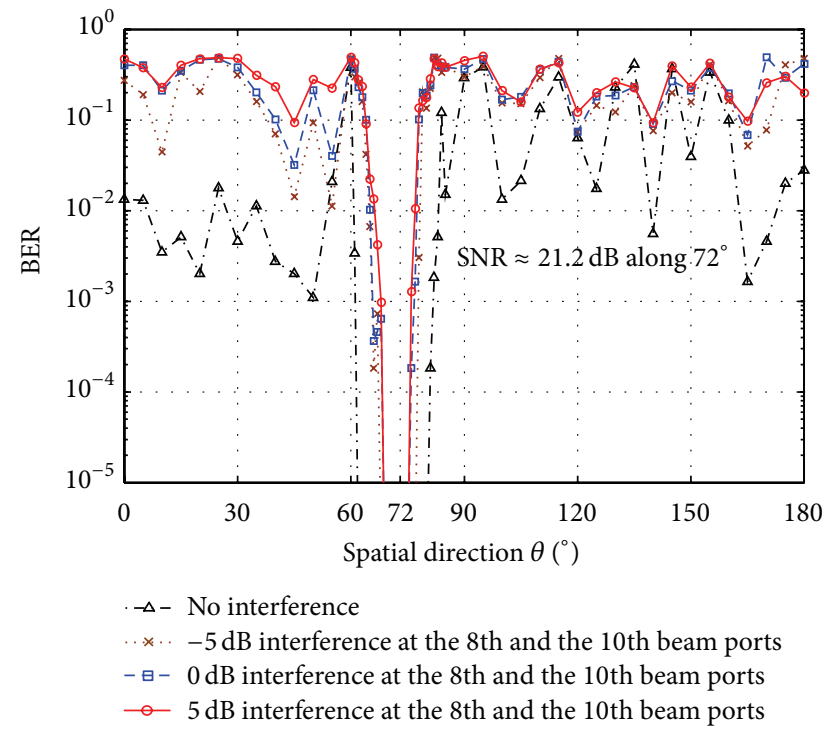

(b)

FIGURE 14: Measured BER spatial distributions for the Fourier Rotman lens, (a) DQPSK and (b) DBPSK DM systems for various interference configurations. BER results for the corresponding conventional beam-steering system are also provided. The SNR for each case was set to $25.8 \mathrm{~dB}$ and $21.2 \mathrm{~dB}$.

Figures $15(\mathrm{~b}), 15(\mathrm{c})$, and $15(\mathrm{~d})$, whose radius is determined by the interference power level. With increased interference power, these circles eventually intersect each other. This greatly obscures the constellation patterns and eventually hinders successful data recovery. Similar constellation patterns, but with 2 symbol states, were obtained for the DBPSK systems. These are omitted for brevity.
3.4. Dual-Beam DM Systems. We now consider that two independent data streams are applied at two different beam ports of the Fourier Rotman lens simultaneously, for example, a DQPSK signal at the 7th beam port and a DBPSK signal at the 9 th beam port. Figure 16 presents the measured BER results for the dual-beam DQPSK-DBPSK DM system. In the experiment the two independent data stream signals used 


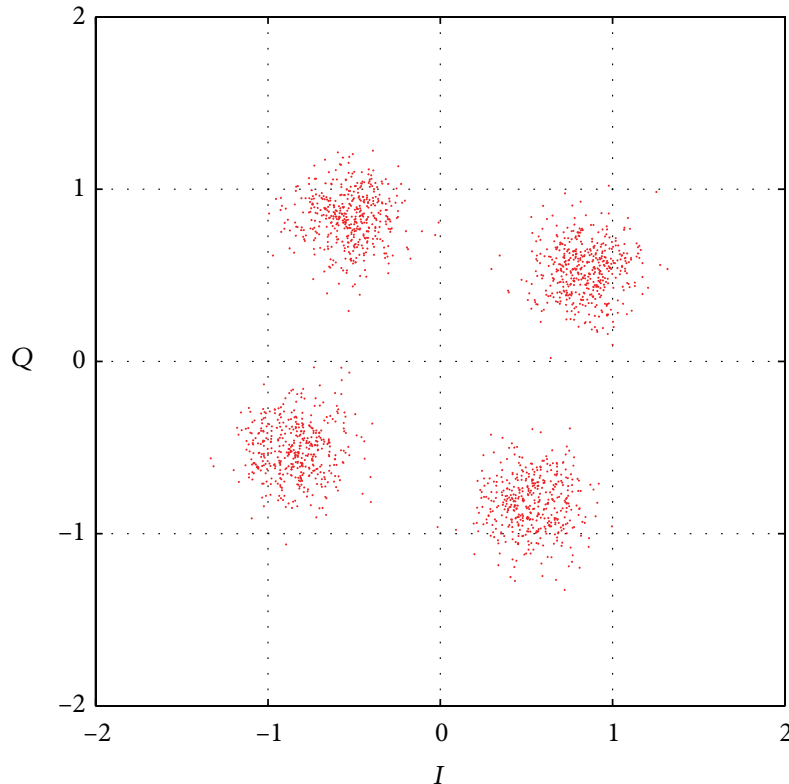

(a)

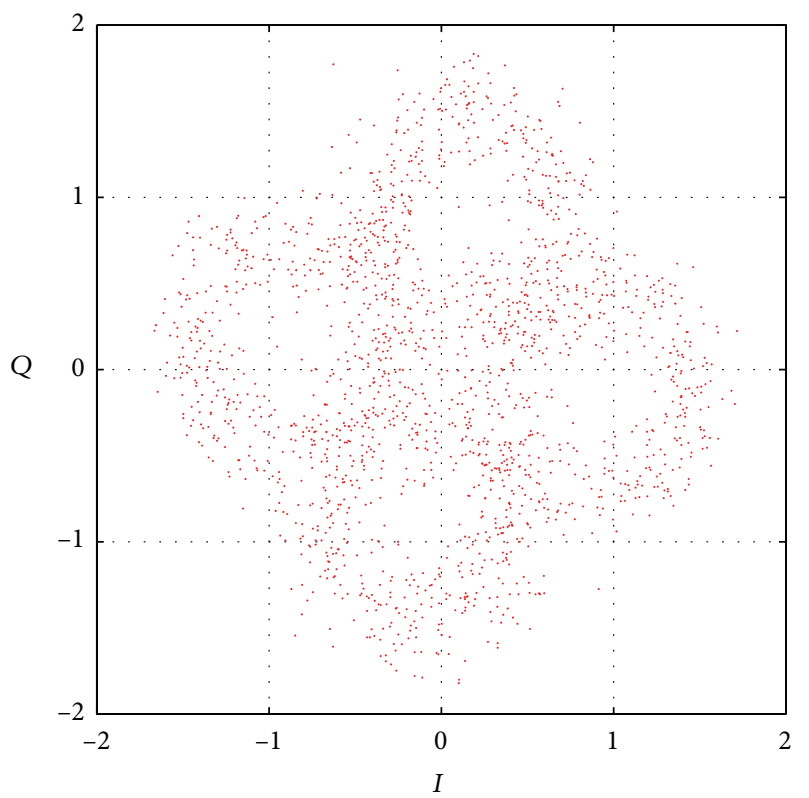

(c)

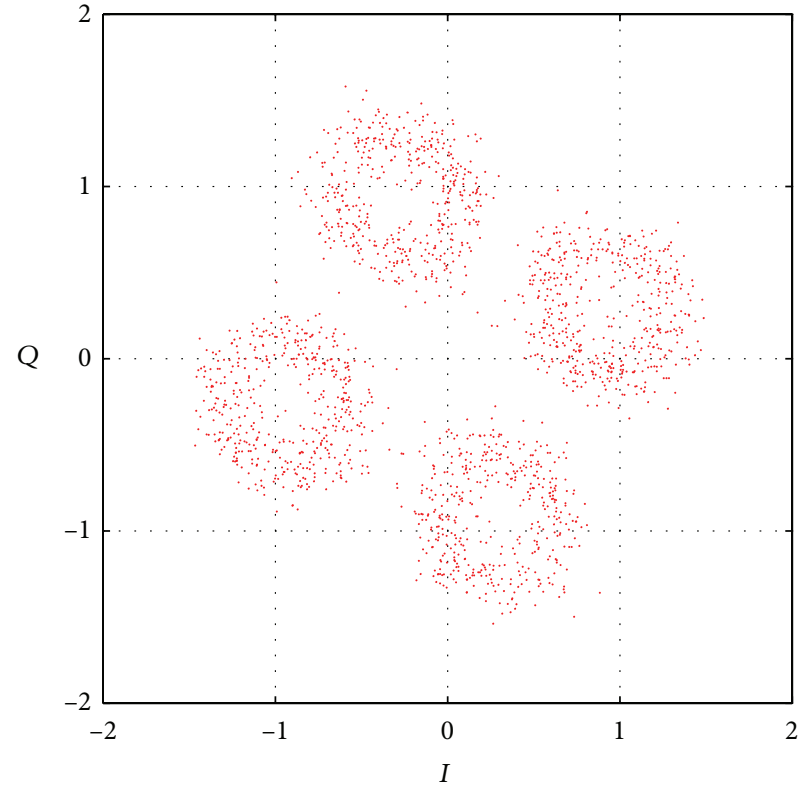

(b)

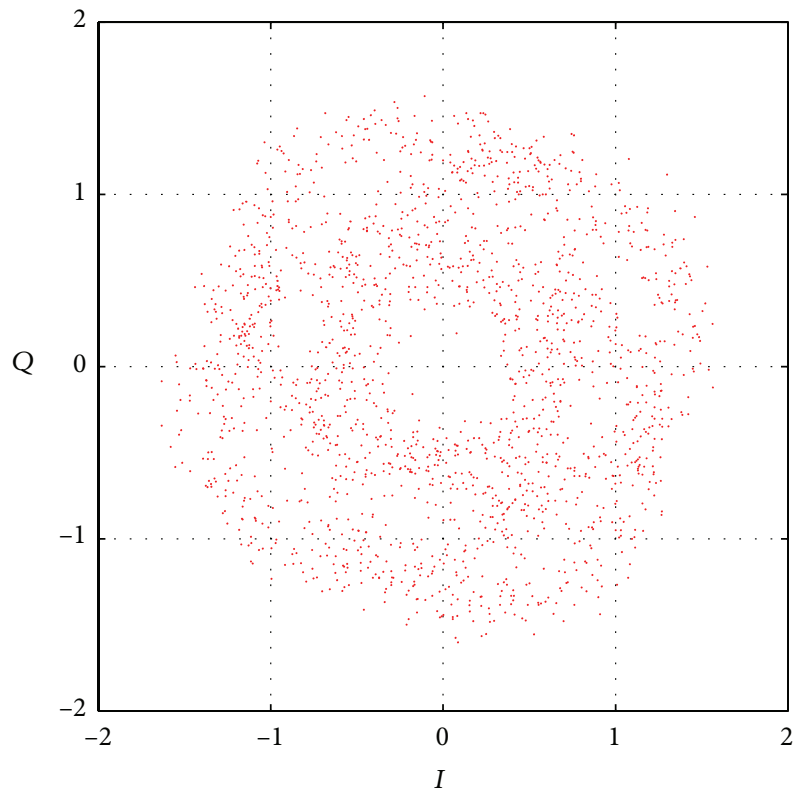

(d)

FIGURE 15: Normalized received constellation diagrams for the DQPSK system with (a) no interference, (b) $-5 \mathrm{~dB}$ interference at the 6 th and the 8 th beam ports, (c) $0 \mathrm{~dB}$ interference at the $6 \mathrm{th}$ and the 8 th beam ports, and (d) $5 \mathrm{~dB}$ interference at the 6 th and the 8 th beam ports along $122^{\circ}$. The DQPSK data stream with $0 \mathrm{~dB}$ power was applied at the 7 th beam port. The SNR along boresight was set to $25.8 \mathrm{~dB}$.

were kept at the same power strength, but we could choose different symbol rates. For comparison, the BER responses in the corresponding conventional DQPSK and DBPSK systems are also provided in Figure 16. In the dual-beam DM systems, the radiated DBPSK signals act as orthogonal interference to the DQPSK signals and vice versa. By setting different symbol rates for the DQPSK and the DBPSK data streams, higher BER values within unselected spatial regions were returned, which may be caused by preamble distortion. It should be noted that both DQPSK and DBPSK data were appended with the same Barker preamble sequence modulated with BPSK, but different symbol rates made them interfere each other. This system is a dynamic DM system, but unlike the previous dynamic ones the interference can only be updated with a limited number of states, for example, two (or four) interference states, corresponding to the number of distinct DBPSK (or DQPSK) symbols, for the DQPSK (or DBPSK) information signals. This can be observed in the received 


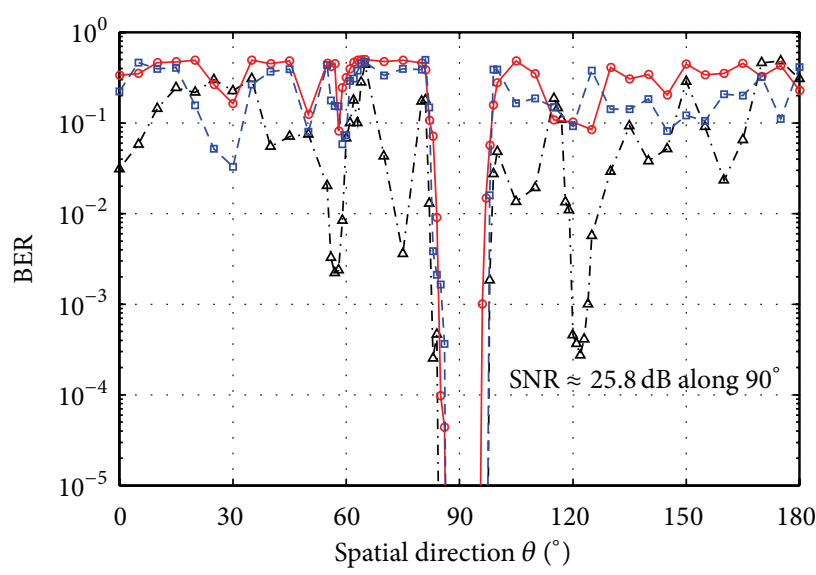

$\triangle-$ No DBPSK signals at the 9th beam port

$-\Xi-$ DBPSK signals with the same power at the 9th beam port, symbol rate of $2.5 \mathrm{Msps}$ for both DQPSK and DBPSK signals

- DBPSK signals with the same power at the 9th beam port, symbol rates of 2.5 Msps for DQPSK and 5 Msps for DBPSK signals

(a)

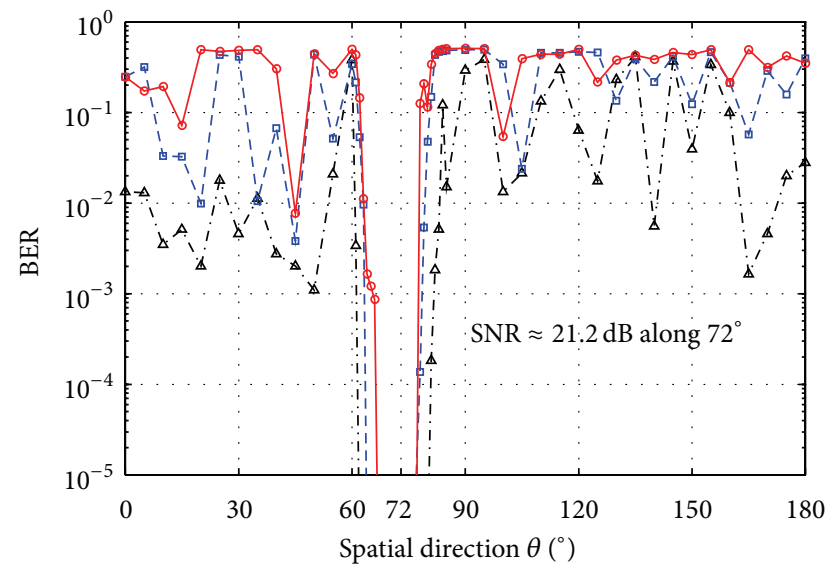

$\rightarrow-$ No DQPSK signals at the 9th beam port

$-{ }_{-}$DQPSK signals with the same power at the 7th beam port, symbol rate of 2.5 Msps for both DQPSK and DBPSK signals

$\rightarrow$ DQPSK signals with the same power at the 7th beam port, symbol rates of 2.5 Msps for DQPSK and 5 Msps for DBPSK signals

(b)

FIGURE 16: Measured BER results for (a) the DQPSK and (b) the DBPSK type receivers in the dual-beam DM systems. The DQPSK signals and the DBPSK signals with the same power were applied at the 7th and the 9th beam ports of the Fourier Rotman lens, respectively. SNR for each receiver case was set to $25.8 \mathrm{~dB}$ and $21.2 \mathrm{~dB}$. The BER responses in the corresponding conventional single-beam DQPSK and DBPSK non-DM systems are also provided.

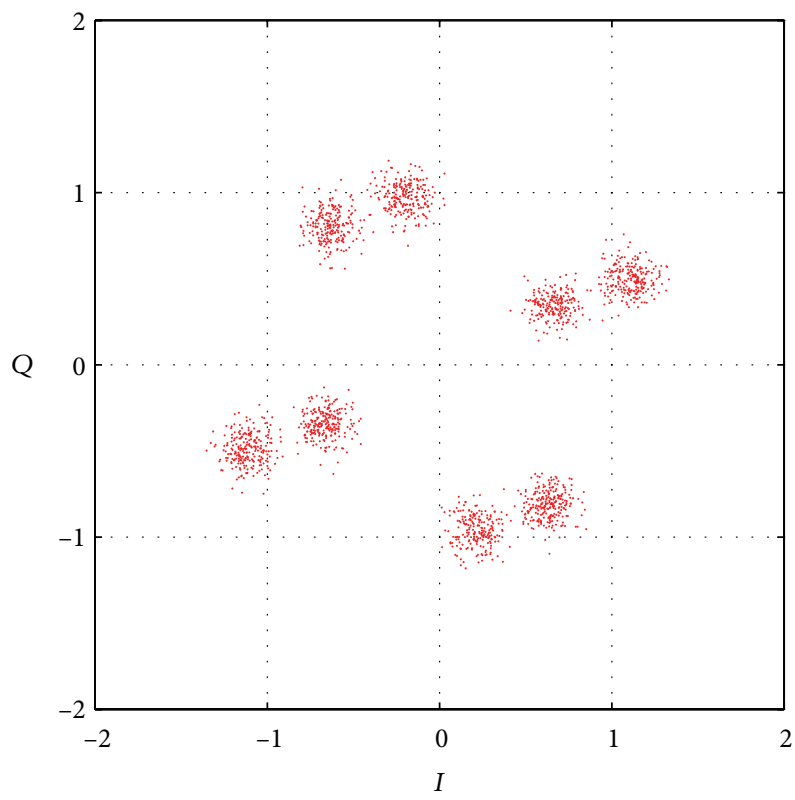

(a)

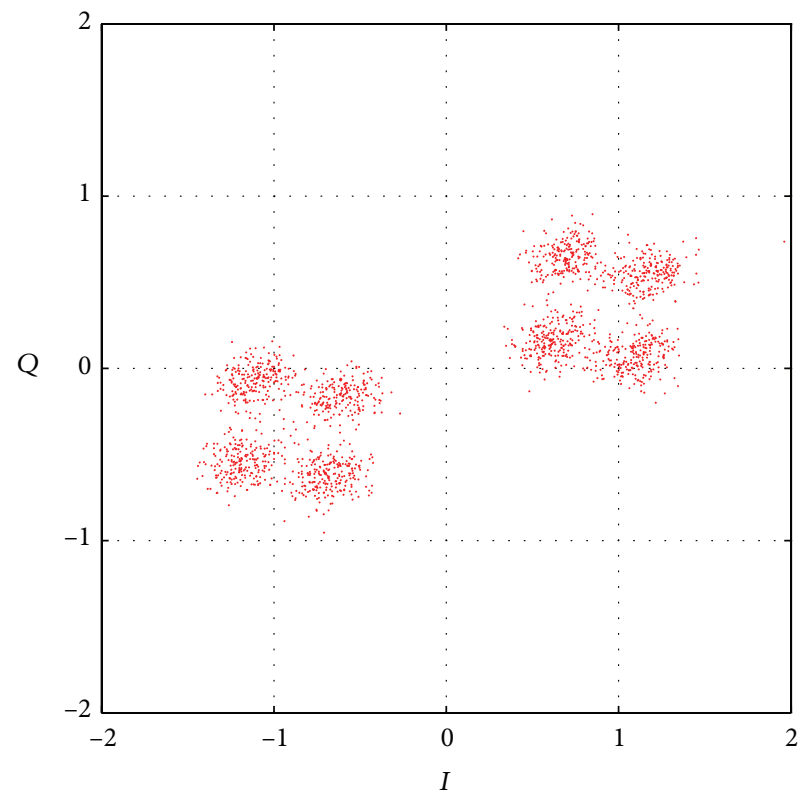

(b)

FIgURE 17: Normalized received constellations diagrams in IQ space along directions of (a) $85^{\circ}$ and (b) $67^{\circ}$. The symbol rates for both the DQPSK and DBPSK data streams were set to $2.5 \mathrm{Msps}$. SNR for each receiver case was set to $25.8 \mathrm{~dB}$ or $21.2 \mathrm{~dB}$ along their desired communication directions, that is, $90^{\circ}$ or $72^{\circ}$.

constellations diagrams in IQ space along directions of $85^{\circ}$ and $67^{\circ}$, respectively, $5^{\circ}$ away from the each specified secure communication direction; see Figure 17.

In order to further distort the constellation patterns along unselected communication directions in a randomized fashion, the remaining 11 beam ports of the Fourier Rotman lens can be excited with random interference. Measured BER results are shown in Figure 18. Again the same interference was applied at the 11 beam ports, and the magnitude of the interference was kept constant during the entire transmission 


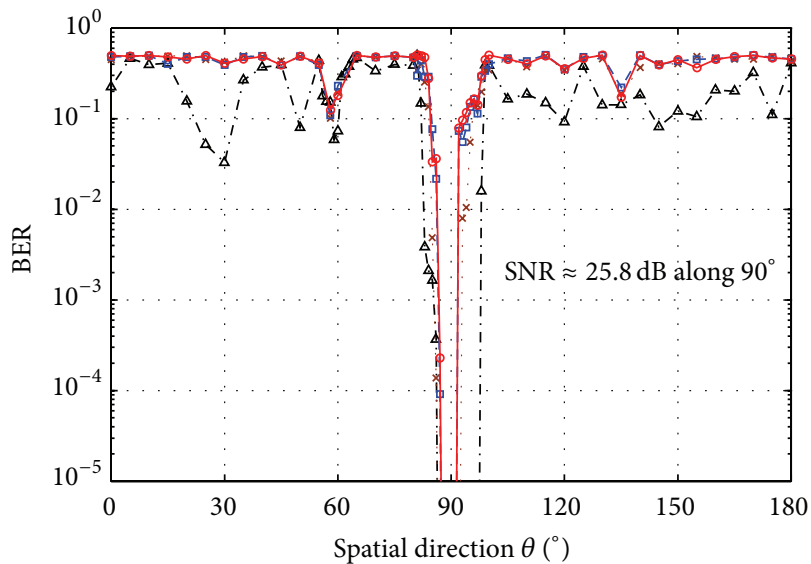

$-\triangle-$ DBPSK signals with the same power at the 9 th beam port, no additional interference

$\ldots *$.. DBPSK signals with the same power at the 9 th beam port, $-15 \mathrm{~dB}$ interference at each of the remaining 11 beam ports

$-\boxminus-$ DBPSK signals with the same power at the 9th beam port, $-10 \mathrm{~dB}$ interference at each of the remaining 11 beam ports

- DBPSK signals with the same power at the 9 th beam port, $-5 \mathrm{~dB}$ interference at each of the remaining 11 beam ports

(a)

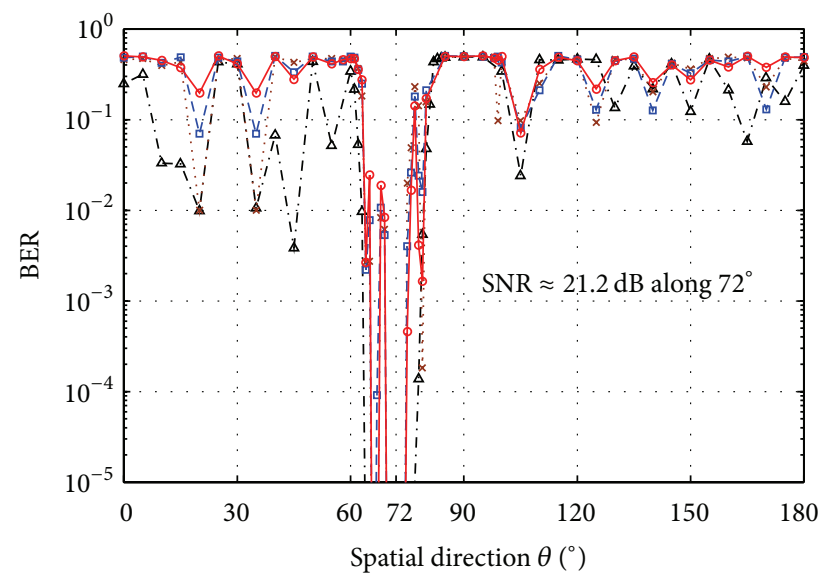

$\Delta-$ DQPSK signals with the same power at the 7 th beam port, no additional interference

$*$.. DQPSK signals with the same power at the 7 th beam port, $-15 \mathrm{~dB}$ interference at each of the remaining 11 beam ports

$-{ }_{-}-$DQPSK signals with the same power at the 7 th beam port, $-10 \mathrm{~dB}$ interference at each of the remaining 11 beam ports

- DQPSK signals with the same power at the 7 th beam port, $-5 \mathrm{~dB}$ interference at each of the remaining 11 beam ports

(b)

FIGURE 18: Measured BER results for (a) the DQPSK and (b) the DBPSK type receivers in the dual-beam DM systems. The DQPSK signals and the DBPSK signals with the same power were applied at the 7th and the 9th beam ports of the Fourier Rotman lens, respectively. The same interference excited the remaining 11 beam ports. SNR for each receiver case was set to $25.8 \mathrm{~dB}$ or $21.2 \mathrm{~dB}$.

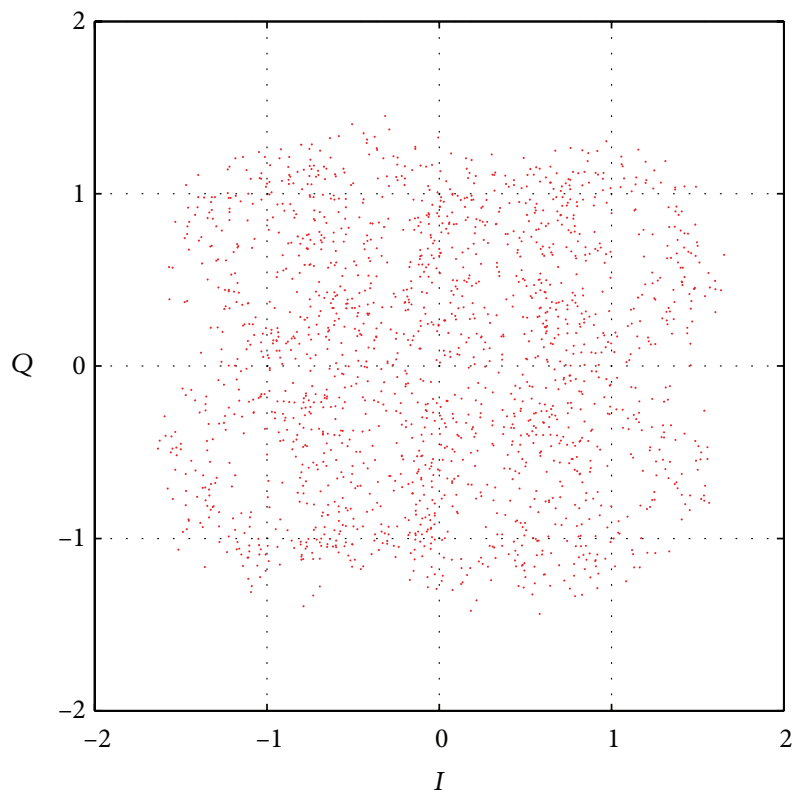

(a)

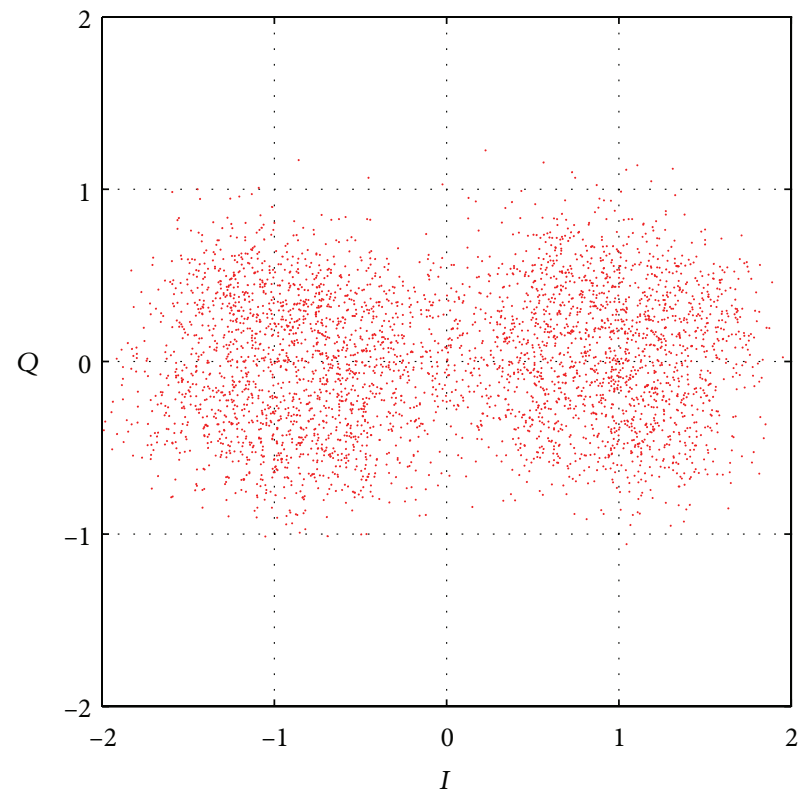

(b)

FIGURE 19: The detected constellation patterns along (a) $85^{\circ}$ and (b) $67^{\circ}$ in the dual-beam DM systems when the (a) DQPSK and (b) DBPSK type receivers were equipped. The DQPSK signals and the DBPSK signals with the same power were applied at the 7th and the 9th beam ports of the Fourier Rotman lens, respectively. The same interference with $-10 \mathrm{~dB}$ power excited the remaining 11 beam ports. SNR for each receiver case was set to $25.8 \mathrm{~dB}$ or $21.2 \mathrm{~dB}$ along their desired communication directions, that is, $90^{\circ}$ or $72^{\circ}$. 
but can vary to construct dual-beam DM systems with different $\mathrm{DM}$ power efficiency, $\mathrm{PE}_{\mathrm{DM}}$; see the definition in (25) in [13]. In the experiment, the interference power at each of the remaining 11 beam ports was chosen to be $-15 \mathrm{~dB}$, $-10 \mathrm{~dB}$, and $-5 \mathrm{~dB}$, which correspond to the $\mathrm{PE}_{\mathrm{DM}} \mathrm{s}$ of $42.6 \%$, $32.2 \%$, and $18.3 \%$. It is reiterated here that in the dualbeam DM systems one information signal acts as interference to the other information signal. Thus, the $\mathrm{PE}_{\mathrm{DM}}$ is always less than $50 \%$ when the two information signals have the same power and additional interference is applied. The phase of the interference is randomly updated within $0^{\circ}$ to $360^{\circ}$ range. The symbol rates for the DQPSK and the DBPSK data streams were set to be identical, $2.5 \mathrm{Msps}$. From the detected constellation patterns along $85^{\circ}$ and $67^{\circ}$ shown in Figure 19, it can be seen that the additional interference spreads each symbol cluster, illustrated in Figure 17, and ultimately leads to higher BER values.

\section{Conclusions}

In this paper, a Fourier Rotman lens DM transmitter for $10 \mathrm{GHz}$ operation has been built and tested for various system configurations. To the authors' best knowledge, it is the first dynamic DM transmitter for real-time data transmission that has been reported. Its successful implementation paves a way for practical yet simple field application of DM technology. Through measured BER spatial distributions and detected constellation diagrams in IQ space, the superiority of the DM transmitter arrays, in terms of directional aware spatial beam confinement performance, over conventional beamsteering arrays has been observed and confirms theoretical simulation.

\section{Conflict of Interests}

The authors declare that there is no conflict of interests regarding the publication of this paper.

\section{Acknowledgments}

This work was sponsored by the Queen's University of Belfast High Frequency Research Scholarship. The authors thank Mr. Michael Major for the fabrication of the Fourier Rotman lens and the antenna array. The authors also would like to thank Mr. Junqing Zhang and Mr. Yuanrui Zhang for useful discussions on WARP hardware.

\section{References}

[1] A. Babakhani, D. B. Rutledge, and A. Hajimiri, "Transmitter architectures based on near-field direct antenna modulation," IEEE Journal of Solid-State Circuits, vol. 43, no. 12, pp. 26742692, 2008.

[2] A. Babakhani, D. Rutledge, and A. Hajimiri, "Near-field direct antenna modulation," IEEE Microwave Magazine, vol. 10, no. 1, pp. 36-46, 2009.

[3] A. H. Chang, A. Babakhani, and A. Hajimiri, "Near-field direct antenna modulation (NFDAM) transmitter at $2.4 \mathrm{GHz}$," in Proceedings of the IEEE Antennas and Propagation Society
International Symposium (APSURSI '09), pp. 1-4, Charleston, SC, USA, June 2009.

[4] M. P. Daly and J. T. Bernhard, "Directional modulation technique for phased arrays," IEEE Transactions on Antennas and Propagation, vol. 57, no. 9, pp. 2633-2640, 2009.

[5] M. P. Daly and J. T. Bernhard, "Beamsteering in pattern reconfigurable arrays using directional modulation," IEEE Transactions on Antennas and Propagation, vol. 58, no. 7, pp. 2259-2265, 2010.

[6] M. P. Daly, E. L. Daly, and J. T. Bernhard, "Demonstration of directional modulation using a phased array," IEEE Transactions on Antennas and Propagation, vol. 58, no. 5, pp. 1545-1550, 2010.

[7] H. Shi and A. Tennant, "Direction dependent antenna modulation using a two element array," in Proceedings of the 5th European Conference on Antennas and Propagation (EUCAP '11), pp. 812-815, IEEE, Rome, Italy, April 2011.

[8] H. Z. Shi and A. Tennant, "An experimental two element array configured for directional antenna modulation," in Proceedings of the 6th European Conference on Antennas and Propagation (EuCAP '12), pp. 1624-1626, IEEE, Prague, Czech Republic, March 2012.

[9] H. Shi and A. Tennant, "Enhancing the security of communication via directly modulated antenna arrays," IET Microwaves, Antennas \& Propagation, vol. 7, no. 8, pp. 606-611, 2013.

[10] T. Hong, M.-Z. Song, and Y. Liu, "Dual-beam directional modulation technique for physical-layer secure communication," IEEE Antennas and Wireless Propagation Letters, vol. 10, pp. 1417-1420, 2011.

[11] O. N. Alrabadi and G. F. Pedersen, "Directional space-time modulation: a novel approach for secured wireless communication," in Proceedings of the IEEE International Conference on Communications (ICC '12), pp. 3554-3558, June 2012.

[12] Y. Ding and V. Fusco, "Vector representation of directional modulation transmitters," in Proceedings of the 8th European Conference on Antennas and Propagation (EuCAP '14), pp. 367371, The Hague, Netherlands, April 2014.

[13] Y. Ding and V. F. Fusco, "A vector approach for the analysis and synthesis of directional modulation transmitters," IEEE Transactions on Antennas and Propagation, vol. 62, no. 1, pp. 361-370, 2014.

[14] Y. Ding and V. F. Fusco, "Establishing metrics for assessing the performance of directional modulation systems," IEEE Transactions on Antennas and Propagation, vol. 62, no. 5, pp. 27452755, 2014.

[15] Y. Ding and V. Fusco, "BER-driven synthesis for directional modulation secured wireless communication," International Journal of Microwave and Wireless Technologies, vol. 6, no. 2, pp. 139-149, 2014.

[16] Y. Ding and V. Fusco, "Directional modulation transmitter synthesis using particle swarm optimization," in Proceedings of the Loughborough Antennas and Propagation Conference (LAPC '13), pp. 500-503, Loughborough, UK, November 2013.

[17] Y. Ding and V. Fusco, "Directional modulation transmitter radiation pattern considerations," IET Microwaves, Antennas and Propagation, vol. 7, no. 15, pp. 1201-1206, 2013.

[18] Y. Ding and V. Fusco, "Constraining directional modulation transmitter radiation patterns," IET Microwaves, Antennas \& Propagation, vol. 8, no. 15, pp. 1408-1415, 2014.

[19] Y. Ding and V. Fusco, "Directional modulation far-field pattern separation synthesis approach," IET Microwaves, Antennas \& Propagation, vol. 9, no. 1, pp. 41-48, 2015. 
[20] Q. Zhu, S. Yang, R. Yao, and Z. Nie, "A directional modulation technique for secure communication based on 4D antenna arrays," in Proceedings of the 7th European Conference on Antennas and Propagation (EuCAP '13), pp. 125-127, Gothenburg, Sweden, April 2013.

[21] Q. Zhu, S. Yang, R. Yao, and Z. Nie, "Directional modulation based on 4-D antenna arrays," IEEE Transactions on Antennas and Propagation, vol. 62, no. 2, pp. 621-628, 2014.

[22] T. Hong, M.-Z. Song, and Y. Liu, "RF directional modulation technique using a switched antenna array for physical layer secure communication applications," Progress in Electromagnetics Research, vol. 116, pp. 363-379, 2011.

[23] N. Valliappan, A. Lozano, and R. W. Heath Jr., "Antenna subset modulation for secure millimeter-wave wireless communication," IEEE Transactions on Communications, vol. 61, no. 8, pp. 3231-3245, 2013.

[24] Y. Zhang, Y. Ding, and V. Fusco, "Sidelobe modulation scrambling transmitter using fourier rotman lens," IEEE Transactions on Antennas and Propagation, vol. 61, no. 7, pp. 3900-3904, 2013.

[25] Y. Ding and V. Fusco, "Sidelobe manipulation using Butler matrix for $60 \mathrm{GHz}$ physical layer secure wireless communication," in Proceedings of the Loughborough Antennas and Propagation Conference (LAPC '13), pp. 61-65, IEEE, Loughborough, UK, November 2013.

[26] Y. Zhang and V. Fusco, "Fourier transform using a Rotman lens," in Proceedings of the 9th European Radar Conference (EuRAD '12), pp. 389-392, November 2012.

[27] W. Rotman and R. Turner, "Wide-angle microwave lens for line source applications," IEEE Transactions on Antennas and Propagation, vol. 11, no. 6, pp. 623-632, 1963.

[28] J. Butler and R. Lowe, "Beam-forming matrix simplifies design of electrically scanned antennas," Electronic Design, vol. 9, pp. 170-173, 1961.

[29] J. Shelton, "Fast Fourier transforms and Butler matrices," Proceedings of the IEEE, vol. 56, no. 3, pp. 350-350, 1968.

[30] M. Ueno, "A systematic design formulation for Butler matrix applied FFT algorithm," IEEE Transactions on Antennas and Propagation, vol. 29, no. 3, pp. 496-501, 1981.

[31] "Fourier Rotman lens directional modulation demonstrator experiment," (Online), https://www.youtube.com/watch?v= FsmCcxo-TPE.

[32] WARP Project, (Online), http://www.warpproject.org.

[33] MATLAB Release, The MathWorks, Natick, Mass, USA, 2013.

[34] Maxim Integrated, "MAX2828/MAX2829 datasheet," http:// www.maximintegrated.com.

[35] Hittite Microwave Corporation, "HMC220AMS8E datasheet," http://www.hittite.com.

[36] "Taconic Advanced Dielectric Division, RF-60A datasheet," (Online), http://www.taconic-add.com/pdf/rf60a.pdf.

[37] MG Chemicals, 500 series FR4 copper clad boards, http://www .mgchemicals.com.

[38] D. M. Pozar, “The active element pattern," IEEE Transactions on Antennas and Propagation, vol. 42, no. 8, pp. 1176-1178, 1994. 

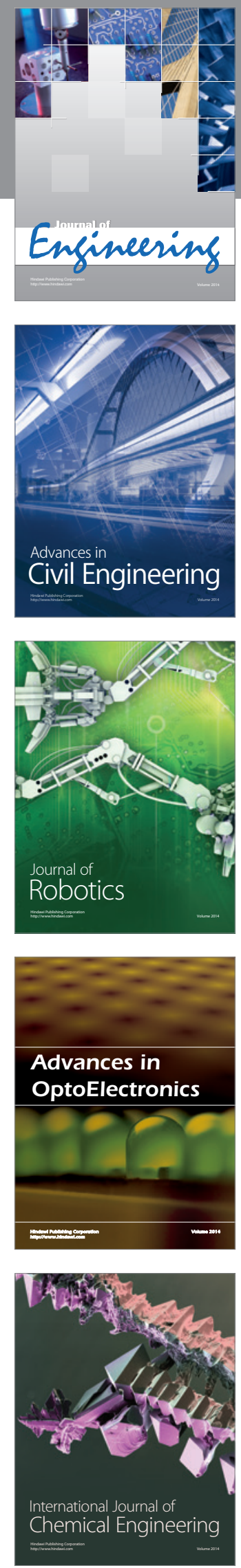

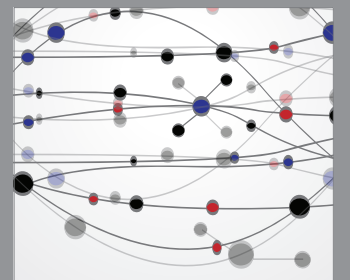

The Scientific World Journal
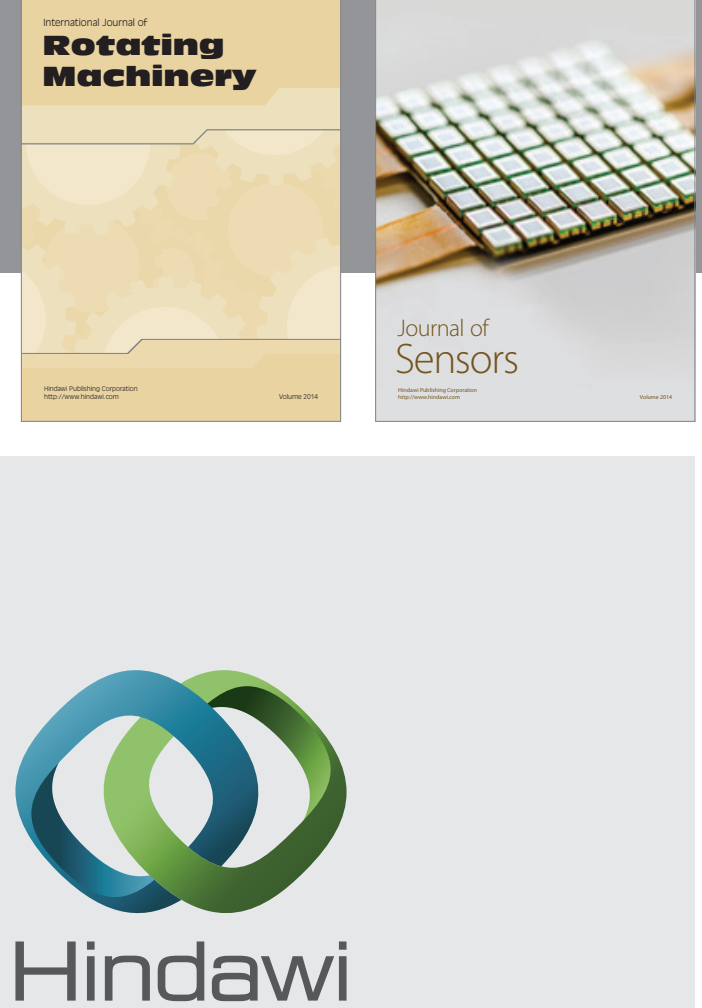

Submit your manuscripts at http://www.hindawi.com
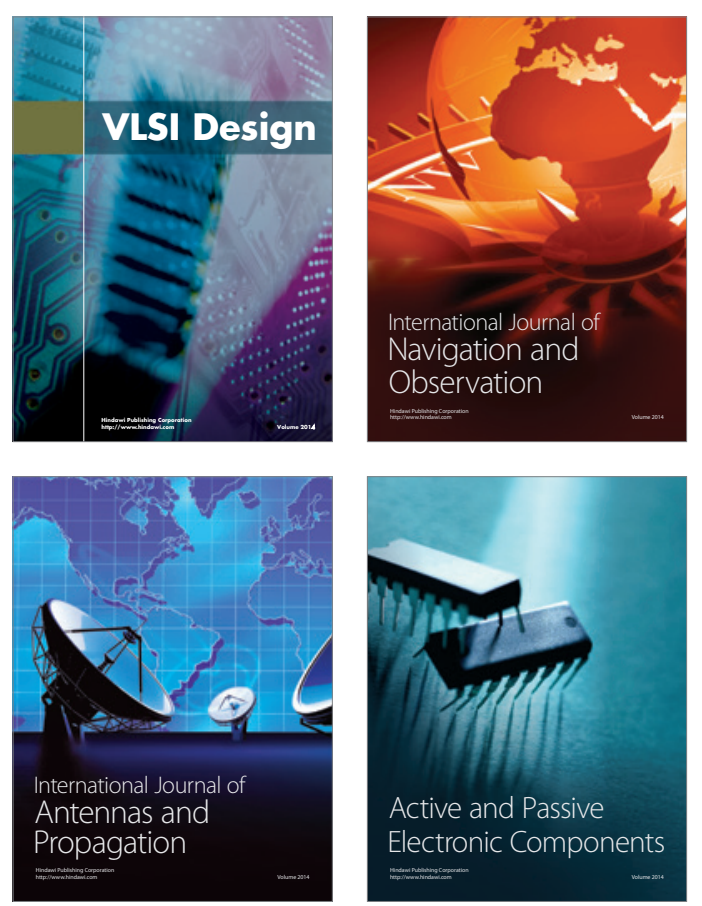
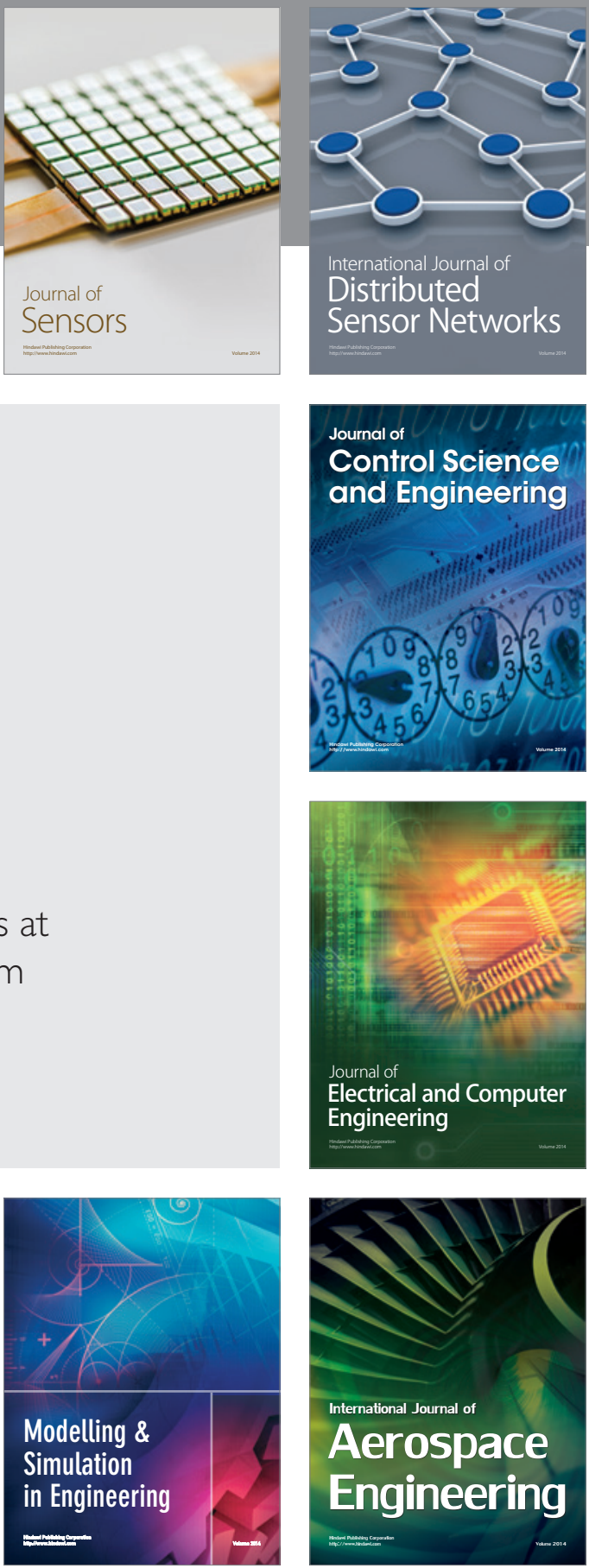

Journal of

Control Science

and Engineering
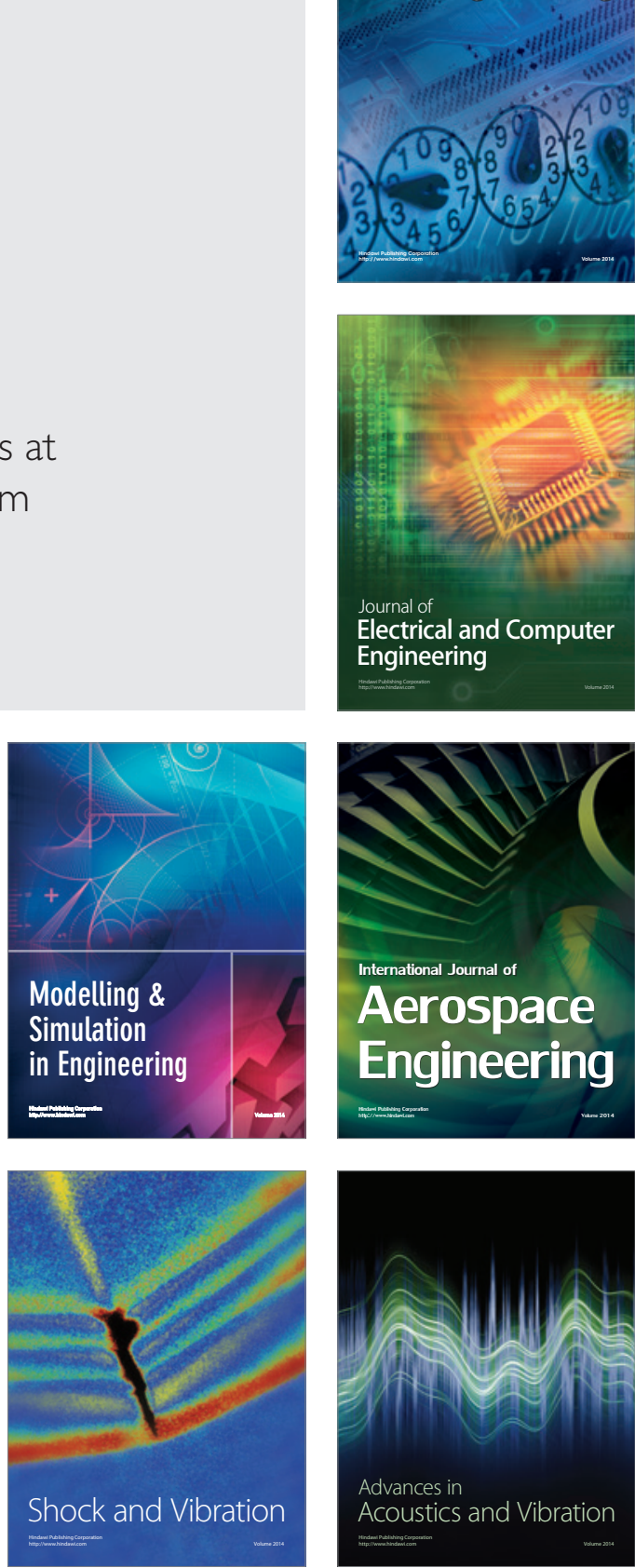